\title{
Análisis técnico económico de la producción de bioetanol a partir de papa a nivel de laboratorio en Boyacá
}

\section{Technical and economic analysis of the production of bioethanol from potatoes in a laboratory in Boyaca}
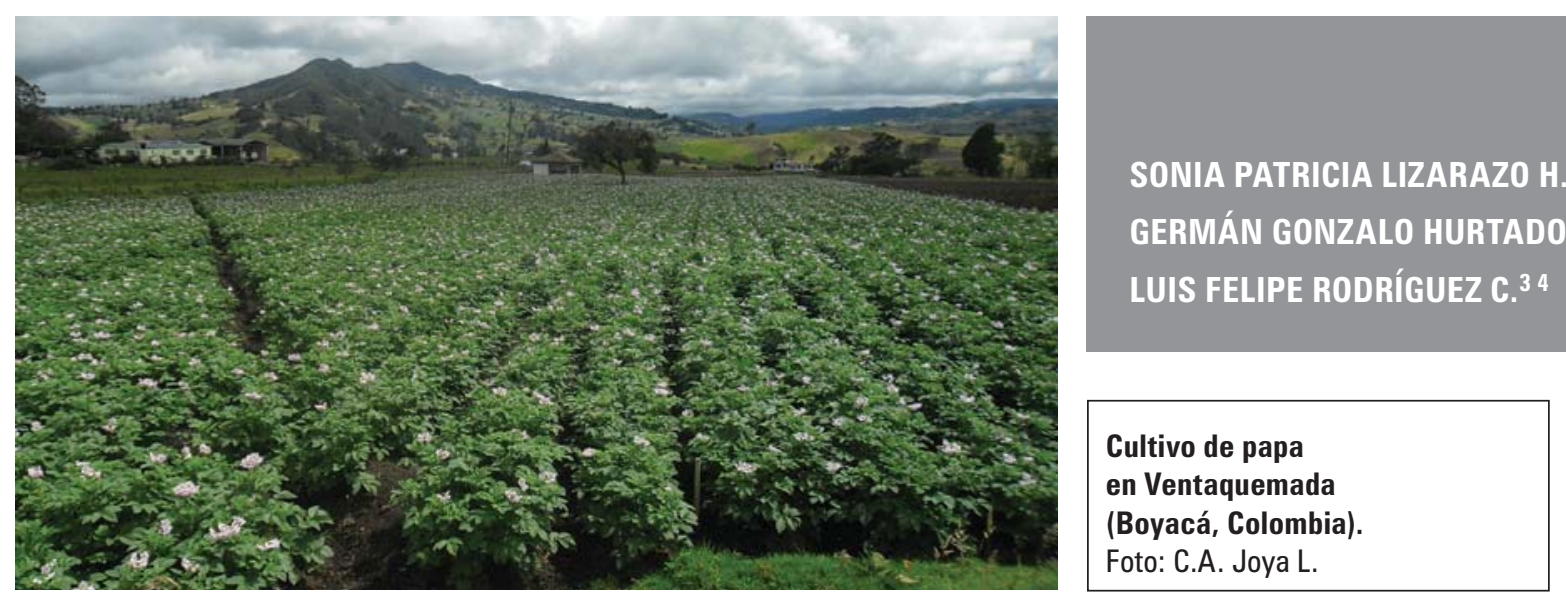

\section{RESUMEN}

El objetivo general de la presente investigación se centró en la evaluación técnica y económica de la producción de etanol como alternativa de uso industrial de las variedades de papa Parda Pastusa y Tuquerreña. Los resultados muestran una eficiencia del $83 \%$ en el proceso de hidrólisis como tratamiento previo al proceso de fermentación. Se determinó que el efecto combinado entre las concentraciones de la enzima $\alpha$-amilasa y el sustrato, se ven favorecidas al utilizar los valores de $0,336 \mathrm{~mL} \mathrm{~kg}^{-1}$ sustrato y $42,2 \%$ respectivamente. La concentración de la enzima amiloglucosidasa y el tiempo de sacarificación no presentaron significancia estadística, por lo que se recomienda utilizar $0,34 \mathrm{~mL} \mathrm{~kg}^{-1}$ sustrato y un tiempo de 14 horas. Para la obtención de alcohol, se requirió un consumo de azúcares reductores de $91 \%$, lo que determinó una densidad del etanol obtenido del $1,014 \mathrm{~g} \mathrm{~cm}^{-3}$ a $20^{\circ} \mathrm{C}$ con una pureza del $94 \%$. Este proceso mostró en la investigación un consumo de azúcares reductores del 41,6\% para el sustrato de la variedad Parda Pastusa y un 75\% para el sustrato de la variedad Tuquerreña, lo cual tiene efecto directo en la producción de alcohol, donde se alcanza un 8,51\% v/v, valor superior al obtenido en el proceso industrial que corresponde a un rango entre 7 y $8 \% \mathrm{v} / \mathrm{v}$. El análisis económico se realiza sobre tres escenarios de inversión, destacándose la materia prima como el rubro más costoso para la producción de etanol.

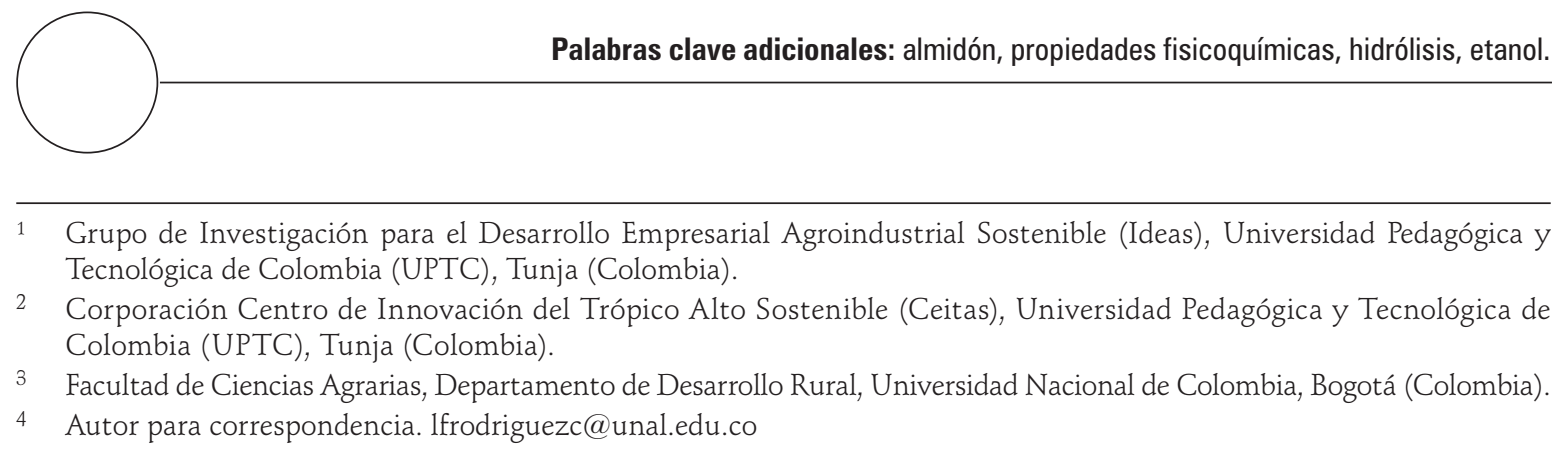




\section{ABSTRACT}

The present study was designed to determine the overall technical and economic potential for the production of ethanol as an alternative use for the potato varieties Tuquerreña and Parda Pastusa. The results showed an efficiency of $83 \%$ in the hydrolysis process as a pretreatment for the fermentation process. It was determined that the combined effect of the concentrations of the $\alpha$-amylase enzyme and substrate was enhanced by using the values $0.36 \mathrm{~mL} \mathrm{~kg}^{-1}$ of substrate and $42.2 \%$, respectively. The concentration of the enzyme amyloglucosidase and the saccharification time did not present any statistical significance, so we recommend using $0.4 \mathrm{~mL} \mathrm{~kg}^{-1}$ of substrate and a time of 14 hours. For the production of alcohol, a consumption of reducing sugars of $91 \%$ was required, resulting in a density of $1.014 \mathrm{~g} \mathrm{~cm}^{-3}$ as obtained from ethanol at $20^{\circ} \mathrm{C}$ with a purity of $94 \%$. This research process showed a consumption of reducing sugars of $41.6 \%$ for the substrate of the Parda Pastusa variety and $75 \%$ for the substrate of the Tuquerreña variety, which had a direct effect on the production of alcohol, which reached $8.51 \% \mathrm{v} / \mathrm{v}$, more than in the industrial process, with a value of between $7 \%$ and $8 \% \mathrm{v} / \mathrm{v}$. The economic analysis was performed on three scenarios of investment, highlighting the raw material as the most expensive cost for producing ethanol.

Additional key words: starch, physicochemical properties, hydrolysis, ethanol.

Fecha de recepción: 10-10-2014

Aprobado para publicación: 28-05-2015
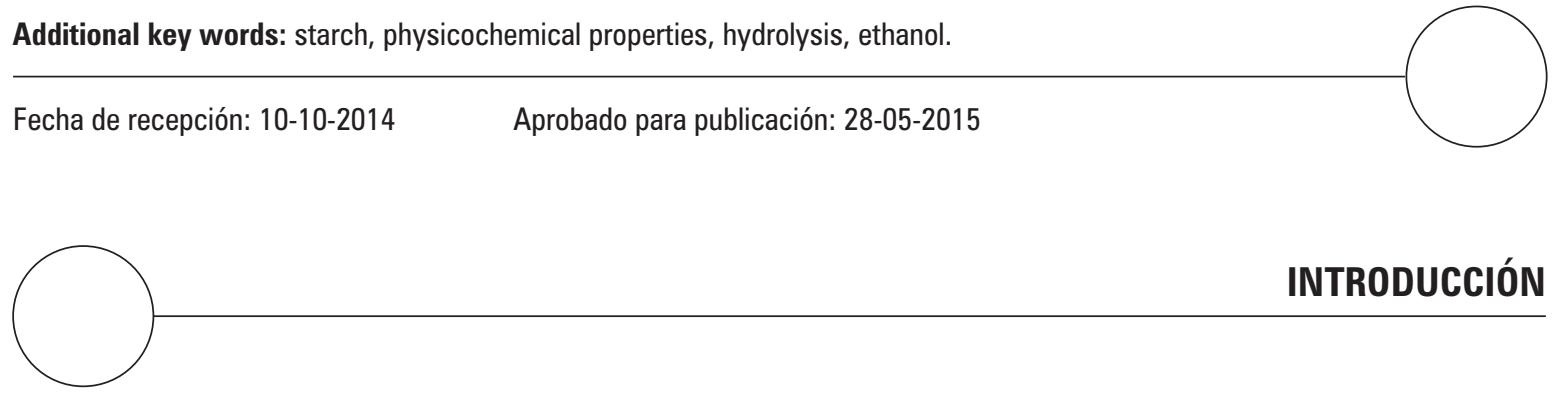

INTRODUCCIÓN

El almidón y su hidrólisis para la producción lo hace materia prima de uso inmediato en procesos alimenticios, industria de lacas, barnices, papel, alimentación animal (Bai et al., 2008). El etanol es uno de los alcoholes de más fácil adquisición y de uso más frecuente en diferentes procesos industriales (Wingrove y Caret, 1981). Se encontró que el uso de la biomasa como fuente de energía es muy competitivo en precio y calidad comparado con los combustibles fósiles, además, empleando biocombustibles se pueden reducir drásticamente las emisiones de $\mathrm{CO}_{2}$ (Santis-Espinosa et al., 2015).

Los tipos de alcoholes que se producen son: alcohol extrapuro, alcohol industrial, alcohol para rones (tafias) y alcoholes superiores (aceite de fúsel). Este producto en la industria licorera del país, en los últimos 10 años ha pre- sentado serios problemas debido al costo de producción, lo cual se ha evidenciado con el cierre de ocho licoreras departamentales en los últimos años (Zuleta y Jaramillo, 2000).

La tecnología para la producción de etanol a partir de materias primas amiláceas involucra tres etapas: (1) licuefacción: proceso hidrolítico en donde los gránulos de almidón se hidratan y se dilatan en condiciones de alta temperatura y donde las enzimas amilolíticas (a-amilasa) produciendo maltodextrinas (Alvis et al., 2008); (2) sacarificación: proceso enzimático en donde enzimas glucoamilolíticas (glucoamilasas o también conocidas como amiloglucosidasas) hidrolizan los enlaces carboxílicos terminales de las moléculas de maltodextrinas a glucosa; (3) fermentación: transformación de azúcares fermentables, pro- 
ducto de la hidrólisis del almidón (maltosa y glucosa), en etanol. Esta transformación se da mediante el uso de la levaduras Saccharomyces cerevisiae (Sánchez et al., 2007).

La producción industrial de bioetanol, a partir de almidón a nivel mundial, emplea diversidad de materias primas: cereales, tubérculos, celulosa entre otros (Castaño et al., 2011). Así mismo, el proceso de obtención de etanol, a partir de estas fuentes, requiere de unas condiciones directamente relacionadas con la composición del almidón, cantidad de amilosa y amilopectina y otros componentes presentes en este. De tal manera, que se asegure una mayor eficacia del tratamiento preliminar, del grado de hidrólisis del almidón, la concentración del azúcar, el pH y de la temperatura óptima de la conversión de almidón a glucosa (Cara et al., 2008), con el fin de obtener una eficiente conversión a etanol.

Según Fedebiocombustibles (2015), la cadena agroindustrial de los biocombustibles en Colombia ha seguido creciendo de una manera importante; con ingeniería nacional se ha ampliado la capacidad instalada de producción de las plantas actuales y se encuentran en producción nuevos proyectos, que incrementarán en, al menos, un $60 \%$ la capacidad instalada actual, para finales de 2014 y finales de 2015, generando o asegurando un porcentaje representativo de empleos.

La papa en la economía de Colombia cumple un rol muy importante, por ser un componente destacado de la canasta familiar y de seguridad alimentaria (Moreno, 2006). Se produce en diferentes departamentos en Colombia, concentrando su mayor producción en Cundinamarca y Boyacá, dada las características climáticas y geográficas de estas zonas (Fedepapa, 2010). Se debe tener en cuenta que el punto de referencia de los cálculos de costos es la producción de etanol, debido a que mayoritariamente el alcohol etílico es transforma- do en alcohol anhídrido debido a la creciente demanda del biocombustible en Colombia; sin embargo, si solo se pretende producir alcohol etílico sin desnaturalizar cabe indicar que los costos son los mismos hasta la primera destilación del proceso del alcohol anhídrido, y puede ser también utilizado en la industria alimentaria, cosmética, farmacológica y producción de bebidas.

Según Nústez (2011), el municipio de Boyacá con la mayor concentración de la producción de papa es Ventaquemada. Según censo efectuado en este departamento en el año 2002, de los 123 municipios de esta unidad territorial en 81 se produce este importante tubérculo. También se destaca el hecho de la evidencia fuerte en Boyacá de una estructura minifundista en este sistema productivo. En esta realidad el cultivo de la papa en Boyacá es de importancia estratégica dado que no solo existe gran tradición sino que se constituye en uno de los sistemas productivos bandera de la economía boyacense.

En contexto de la actividad productiva agraria tanto en el país como en el departamento de Boyacá es necesario promover las buenas prácticas en la agricultura para proteger las fuentes de agua y trabajar por la seguridad alimentaria, por lo que se debe formalizar un compromiso muy fuerte de los productores del sistema productivo papa para que se contribuya a lograr un equilibrio ambiental, máxime cuando este sistema productivo es estratégico de la seguridad alimentaria y como proveedor potencial de materia prima para la producción de bioetanol (Arias, 2014; Lizarazo et al., 2015).

El objetivo de esta investigación se centró en la evaluación técnica y económica de la producción de etanol como alternativa de uso de las variedades de papa Parda Pastusa y Tuquerreña para la industria licorera, evaluando los costos de producción a nivel de laboratorio, utilizando el modelo ABC (Activity Based Costing). 


\section{METODOLOGÍA Y PROCEDIMIENTO DE INVESTIGACIÓN}

El experimento epicentro de esta investigación se llevó a cabo en el laboratorio de control biológico adscrito a la Facultad de Ciencias Agropecuarias de la Universidad Pedagógica y Tecnológica de Colombia (Tunja, Boyacá).

El experimento se realizó empleando dos tratamientos correspondientes a las variedades de papa Parda Pastusa y Tuquerreña (Solanum tuberosum L.), procedentes del departamento de Boyacá, Colombia. En razón a las condiciones ambientales altamente controladas del laboratorio donde se llevó a cabo el experimento, el diseño experimental empleado en el montaje del ensayo fue de carácter no aleatorio y para el análisis técnico económico de la información recolectada se emplearon técnicas de estadística descriptiva tales como media aritmética, error estándar y porcentajes.

Los tubérculos de papa se clasificaron por sanidad, se limpiaron y se retiró manualmente la epidermis. Los almidones se obtuvieron según metodología descrita por Singh y Singh (2001), tomando $1 \mathrm{~kg}$ de cada una de las variedades. Se fraccionó y se colocó el producto en agua destilada con metabisulfito de sodio ( $35 \mathrm{~g} / 100 \mathrm{~L}$ ). Posteriormente se procedió a la extracción del almidón, se lavó varias veces con agua destilada hasta que el líquido efluente fue claro (Singh y Singh, 2001). El almidón se dejó reposar, decantar y se filtró. La pasta resultante se llevó a una estufa de aire caliente a $40^{\circ} \mathrm{C}$ por $24 \mathrm{~h}$. Obtenido el almidón seco, se pasó por un molino pulverizador marca Cemotec ${ }^{\circledR} 1090$ SampleMill (Foss Tecator AB, Höganäs, Suecia), para reducir el tamaño de partícula, se tamizó usando la malla número 100 y finalmente fue almacenado en bolsas con cierre hermético, como se describe en la figura 1.

\section{Preparación de la dispersión de almidón}

Se tomaron $2 \mathrm{~kg}$ de almidón de papa refinado, con una humedad del $10 \%$, y se dispersaron en

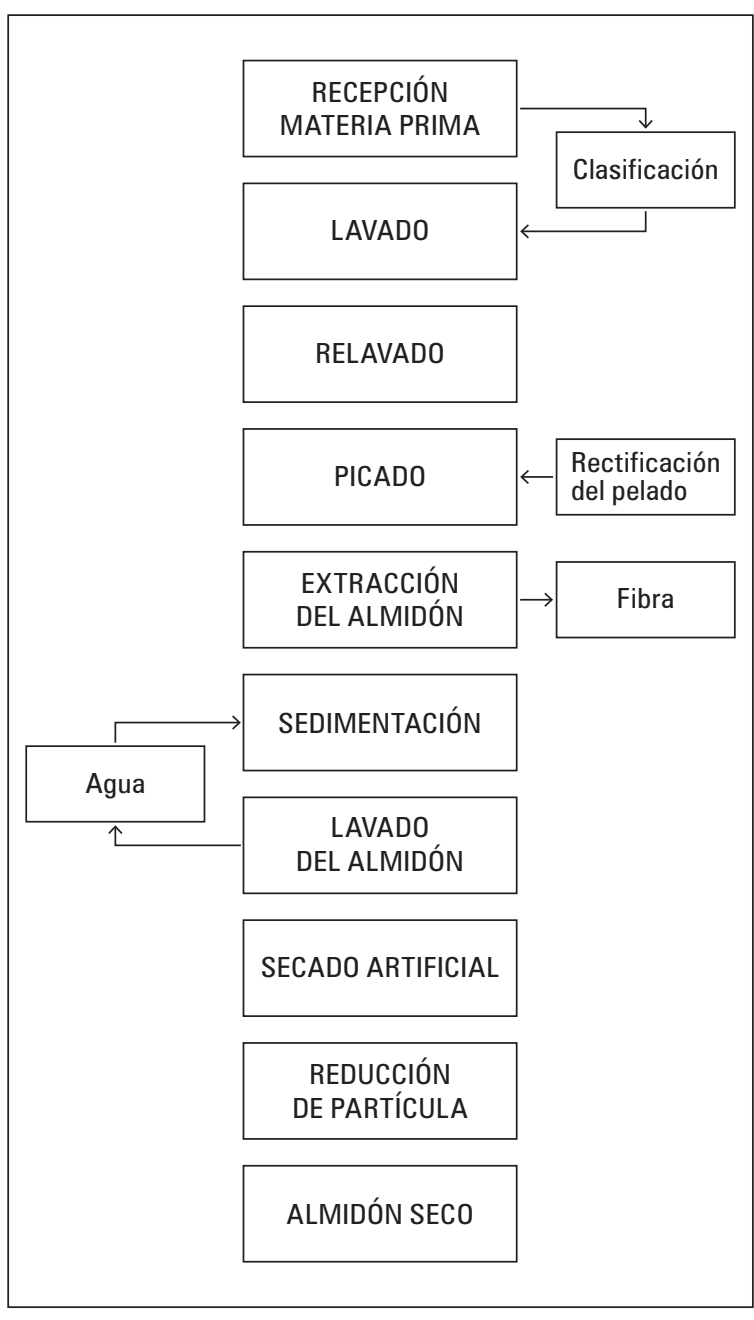

Figura 1. Diagrama de proceso para la obtención de almidón de papa.

frío en 3,5 L de agua destilada, hasta alcanzar un volumen final de $5 \mathrm{~L}$. Se procedió a ajustar el $\mathrm{pH}$ con $\mathrm{HCl} 1 \mathrm{~N}$ hasta un valor de 5,5. Luego se ajustó la concentración de $\mathrm{Ca}^{2+}$ a 30 ppm mediante la adición de $\mathrm{CaCl}_{2} 2 \mathrm{H}_{2} \mathrm{O}$ grado analítico. La mezcla anterior se transfirió a un biorreactor Braun ${ }^{\circledR}$ (Sartorius AG, Göttingen, Alemania) de $5 \mathrm{~L}$, con control de temperatura, $\mathrm{pH}$ y velocidad de agitación con precisión de $0,1^{\circ} \mathrm{C} \pm 0,01$ y 1 rpm, respectivamente.

\section{Licuefacción del almidón}

En el proceso de licuefacción, se empleó $\alpha$-amilasa de Sigma-Aldrich, que es una enzi- 
ma bacteriana proveniente del Aspergillus oryzae. Previamente se determinó la temperatura de licuefacción para las dos variedades seleccionadas. Se realizaron dos ensayos por duplicado basados en el siguiente procedimiento: en un recipiente beaker se pesó una solución con concentración de $42,69 \%$ de almidón y $57,30 \%$ de agua destilada. Teniendo en cuenta las temperaturas y los tiempos, se trabajó la gelatinización por 20 min. Se licuó la muestra dos minutos a velocidad baja y se transfirió a un beaker de $350 \mathrm{~mL}$ donde se corrigió el pH 4,5, con $\mathrm{HCl}$ 0,1 $\mathrm{N}$ y se adicionó la enzima $\alpha$-amilasa. Para la dosificación de la enzima comercial, los cálculos se realizaron de acuerdo con las especificaciones del proveedor, los cuales corresponden a una dosis de $336,6 \mu \mathrm{L}$, a una temperatura de $85^{\circ} \mathrm{C}$ por un tiempo de 90 min, agitación constante y a un pH de 6 .

La cinética de licuefacción se monitoreó a partir del momento en que se alcanzó el equilibrio térmico; se realizó a partir del seguimiento de la concentración de azúcares reductores por el método DNS (ácido 3,5 dinitro salicílico), por espacio de 2 $\mathrm{h}$, a intervalos de $0,5 \mathrm{~h}$ de acuerdo con la metodología reportada por Castaño y Mejía (2008).

\section{Sacarificación enzimática - fermentación independiente}

La etapa de sacarificación se efectuó con la enzima glucoamilasa fúngica de Sigma-Aldrich, obtenida a partir de Aspergillus niger. El proceso se llevó a cabo según las condiciones de temperatura, pH, acidez, ${ }^{\circ}$ Brix establecidas por el proveedor. Concluido el proceso de presacarificación, se ajustó el pH a 4,5 con $\mathrm{HCl} 0,1 \mathrm{~N}$. Para la dosificación de la enzima comercial se tomó como referente las especificaciones del catálogo Sigma-Aldrich. Los cálculos se realizaron con base en almidón seco. De acuerdo con lo anterior, se adicionó $340,6 \mu \mathrm{L}$ de enzima de glucoamilasa al sustrato a una temperatura de $50^{\circ} \mathrm{C}$ con agitación constante. El tiempo como variable se trabajó conforme a los estudios referenciados por Lezame (2006) y se ajustó para el caso. Se tomaron dos muestras de $4 \mathrm{~mL}$ cada una, las que se centrifugaron durante $20 \mathrm{~min}$ a una temperatura de $4^{\circ} \mathrm{C}$ a $500 \mathrm{rpm}$ en los tubos plásticos y posteriormente se analizaron en el espectrofotómetro para medir el contenido de azúcar en las muestras y cuantificar en equivalentes de dextrosa (ED).

\section{Medios de cultivo}

Los medios de cultivo para los inóculos fueron preparados con glucosa $2 \%$, extracto de levadura 0,5\%, $\left(\mathrm{NH}_{4}\right)_{2} \mathrm{SO}_{4} 0,1 \%, \mathrm{KH}_{2} \mathrm{PO}_{4} 0,1 \%, \mathrm{MgSO}_{4}$ $7 \mathrm{H}_{2} \mathrm{O} 0,05 \%$. Se prepararon medios con concentraciones de $20 \mathrm{~g} \mathrm{~L}^{-1}$ de azúcares reductores (Cano y Naranjo, 2002).

\section{Fermentación}

La etapa de fermentación se desarrolló por duplicado, para las dos variedades de papa. Se tomó $775 \mathrm{~g}$ proveniente de la etapa anterior correspondiente a un volumen de $1.500 \mathrm{~mL}$ de jarabe de glucosa. Se licuó por 2 min a baja velocidad. Se procedió a esterilizarlo en autoclave a 15 psi y a una temperatura de $120^{\circ} \mathrm{C}$ por $15 \mathrm{~min}$. Esta solución se diluyó con agua estéril hasta una concentración de $12{ }^{\circ}$ Brix y a una temperatura de $30^{\circ} \mathrm{C}$, inmediatamente se le agregaron $2 \mathrm{~g} \mathrm{~L}^{-1} \mathrm{de}$ $\mathrm{NH}_{4} \mathrm{NO}_{3}, 1 \mathrm{~g} \mathrm{~L}^{-1}$ de $\mathrm{MgSO}_{4}, 1 \mathrm{~g} \mathrm{~L}^{-1}$ de $\mathrm{KH}_{2} \mathrm{PO}_{4}$ y $2 \mathrm{~g} \mathrm{~L}^{-1}$ de extracto de levadura, metodología empleada por González et al., (2006).

Una vez finalizado el proceso de licuefacción, el sistema se llevó a condición de operación de procesos simultáneos (temperatura de $30,0^{\circ} \mathrm{C}$ $\pm 0,1^{\circ} \mathrm{C}$ y a $\mathrm{pH}$ de $\left.4,50 \pm 0,01\right)$, mediante enfriamiento con agua potable a través de la chaqueta del biorreactor y adición de $\mathrm{HCl}$ 1,0 N. El producto de la licuefacción se suplemento con $\left(\mathrm{NH}_{4}\right)_{2}$ $\mathrm{HPO}_{4} 6,0 \mathrm{~g} \mathrm{~L}^{-1}, \mathrm{MgSO}_{4} 7 \mathrm{H}_{2} \mathrm{O} 2 \mathrm{~g} \mathrm{~L}^{-1}$ y $\mathrm{KH}_{2} \mathrm{PO}_{4}$ $3,0 \mathrm{~g} \mathrm{~L}^{-1}$, respectivamente. En ese momento, se adicionó la enzima $\alpha$-amilasa teniendo en cuenta los resultados previos. Después de la adición del enzima, se procedió a la adición del inóculo de la levadura Saccharomces cerevisiae (marca 
Instant Yeast), en un volumen correspondiente al $10 \%$ del volumen útil del biorreactor.

El inóculo se preparó en el hidrolizado de la licuefacción, enriquecido con las sales descritas en la metodología y se activó durante $4 \mathrm{~h}$, a $30,0^{\circ} \mathrm{C} \pm 0,1^{\circ} \mathrm{C}$, empleando un agitador orbital a $110 \pm 1 \mathrm{rpm}$, a partir de la adición de levadura, se determinó la concentración de azúcares reductores mediante lectura de ${ }^{\circ}$ Brix a través del refractómetro y se ajustó para garantizar el proceso de fermentación. La estimación del tiempo empleado para la fermentación fue de $32 \mathrm{~h}$. Una vez terminado el proceso, se inactivó la levadura por centrifugación a 10.000 rpm por 30 min. Así mismo, con el fin de establecer la eficiencia del proceso de la conversión de almidón a alcohol, se midió la concentración de etanol y azucares reductores.

\section{Destilación}

Obtenido el alcohol, se empleó el método de separación por arrastre de vapor para su separación. Por último, se hizo la evaluación económica de la producción de etanol, se identificaron las actividades, la agregación de valor al proceso. Para ello se aplicó un modelo por órdenes de producción, dado que es el tipo que más se ajusta a nivel de laboratorio, siguiendo el proceso como lo referencian Kaplan y Anderson (2004).

El análisis económico del experimento se fundamentó en el cálculo de los costos de producción e ingresos por litro de alcohol, como de la producción de alcohol resultante del procesamiento del $20 \%$ de la producción total de papa. En la determinación de los costos unitarios para el litro de alcohol se utilizó el sistema de costeo ABC (Miranda, 2012).

\section{Análisis económico}

El análisis económico del experimento se complementó con un análisis financiero a través del cual se efectuó la respectiva evaluación financie- ra del proyecto mediante los indicadores de valor presente neto (VPN) y tasa interna de retorno (TIR). Así mismo se determinó el punto de equilibrio para el experimento (Guzmán, 2008).

\section{ANÁLISIS Y DISCUSIÓN DE RESULTADOS}

\section{Condiciones de hidrólisis enzimática para la obtención de jarabe de glucosa}

\section{Temperatura de gelatinización del almidón de papa}

Al ser sometido a calentamiento progresivo y transcurrido un tiempo de 8 min a una temperatura de $57^{\circ} \mathrm{C}$ el almidón de papa de las variedades Parda Pastusa y Tuquerreña, percibe cambios en su viscosidad y transparencia. Estos valores de temperatura están en concordancia con los rangos reportados en la literatura para el almidón nativo de papa, el cual se encuentra entre 56 y $67^{\circ} \mathrm{C}$ (Lideboom et al., 2004).

La gelatinización, según reportan algunos autores, en los almidones de ñame, papa y yuca modificados físicamente por calor húmedo son más fácilmente hidrolizados por la acción del $\alpha$-amilasa que los almidones nativos. Además, la gelatinización del almidón de papa y del arroz incrementa la susceptibilidad a la acción de las enzimas amilolíticas en razón a la proporción de amilosa y amilopectina (Gunaratne y Hoover, 2002).

\section{Pretratamiento del almidón}

La acción del enzima $\alpha$-amilasa agregada al sustrato presentó una mayor actividad a un $\mathrm{pH}$ de 5,3-5,5 en tampón de hidróxido de calcio al $0,1 \%$, dando como resultado un porcentaje de azúcares reductores de 5,98\% para 'Parda Pastusa' y de 3,70\% para 'Tuquerreña' (figura 2). Esta diferencia se debe a que la eficiencia de la $\alpha$-amilasa siempre se va a favorecer más en concentraciones mayores de amilosa. De este resul- 


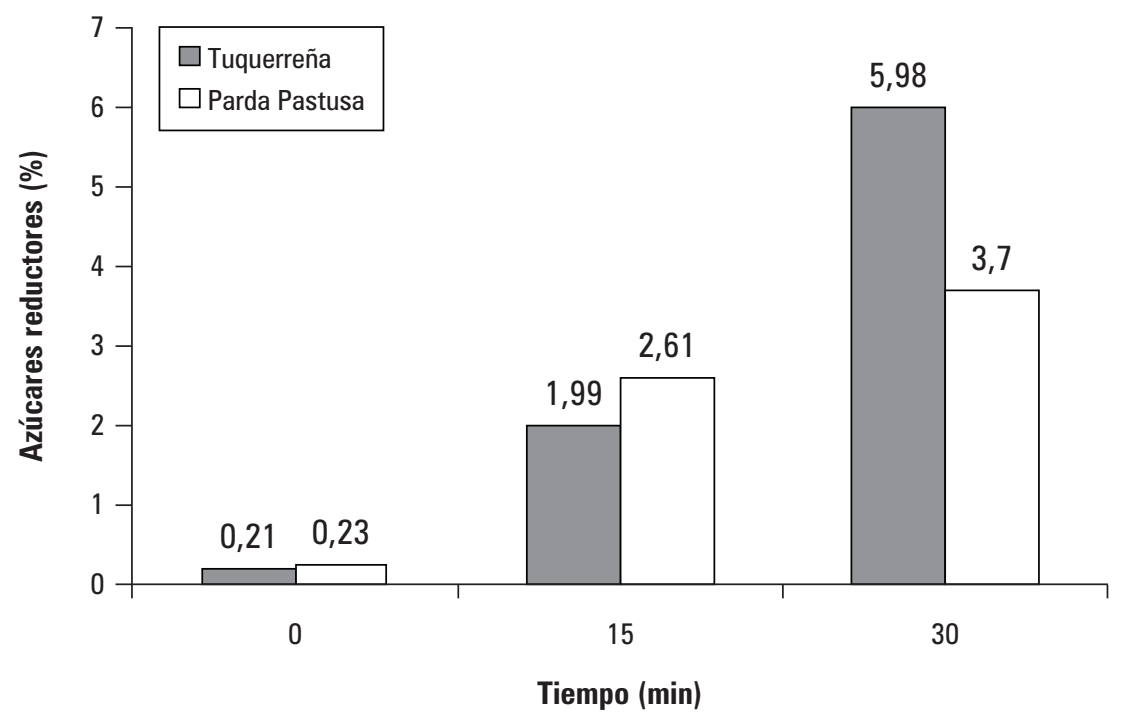

Figura 2. Licuefacción del almidón por acción de la enzima $\alpha$-amilasa.

tado se infiere que es posible que el porcentaje de amilosa sea mayor en la variedad Parda Pastusa que en la Tuquerreña.

\section{Sacarificación del almidón}

Llevado a cabo el proceso de licuefacción es necesario agregar al sustrato parcialmente hidrolizado la enzima glucoamilasa para separar los enlaces 1-6 de la amilopectina. La acción de la enzima glucoamilasa presentó una mayor actividad a un pH de 4,4 a 4,6 en tampón de ácido $\mathrm{HCl}$ al $0,1 \mathrm{~N}$ dando como resultado un porcentaje de azúcares reductores de 13,73\% y 10,60\% para 'Parda Pastusa' y 'Tuquerreña', respectivamente. A partir de este resultado se infiere que la variedad 'Parda Pastusa', si se compara con la variedad Tuquerreña, presentó una mayor eficiencia en el proceso de hidrólisis, debido quizás al contenido de amilopectina, lo cual permite una mayor acción enzimática y, por ende, una mayor concentración de azúcares (figura 3).

De acuerdo con los resultados, se infiere que el mayor porcentaje de azúcares reductores para las dos variedades analizadas, se obtuvo a una concentración de $0,8 \mathrm{~mL} \mathrm{~kg}^{-1}$ sustrato de enzima en solución al 40\% de almidón, empleando un tiempo de hidrólisis para la $\alpha$-amilasa de 0,5 h y para la enzima glucoamilasa de $14 \mathrm{~h}$.

Así mismo, se realizaron análisis previos a la hidrólisis, como la composición química del almidón y el análisis físico e instrumental; a partir de estos se pudo comprobar que en el caso de la $\alpha$-amilasa, esta alcanza una actividad máxima para un $\mathrm{pH}$ de 4,7 y una temperatura eficiente de $55^{\circ} \mathrm{C}$. Para la glucoamilasa, por el contrario, el pH óptimo es de 4,0 tendiendo a 4,7 y una actividad equivalente al 70\% de la actividad máxima, y la temperatura óptima $75^{\circ} \mathrm{C}$, dado que se trata de un enzima inestable a altas temperaturas, el límite para aplicaciones prácticas se halla alrededor de los $60^{\circ} \mathrm{C}$.

\section{Caracterización del jarabe de glucosa}

En la tabla 1 se presenta la composición química de los jarabes de glucosa obtenidos de las variedades objeto de estudio. 


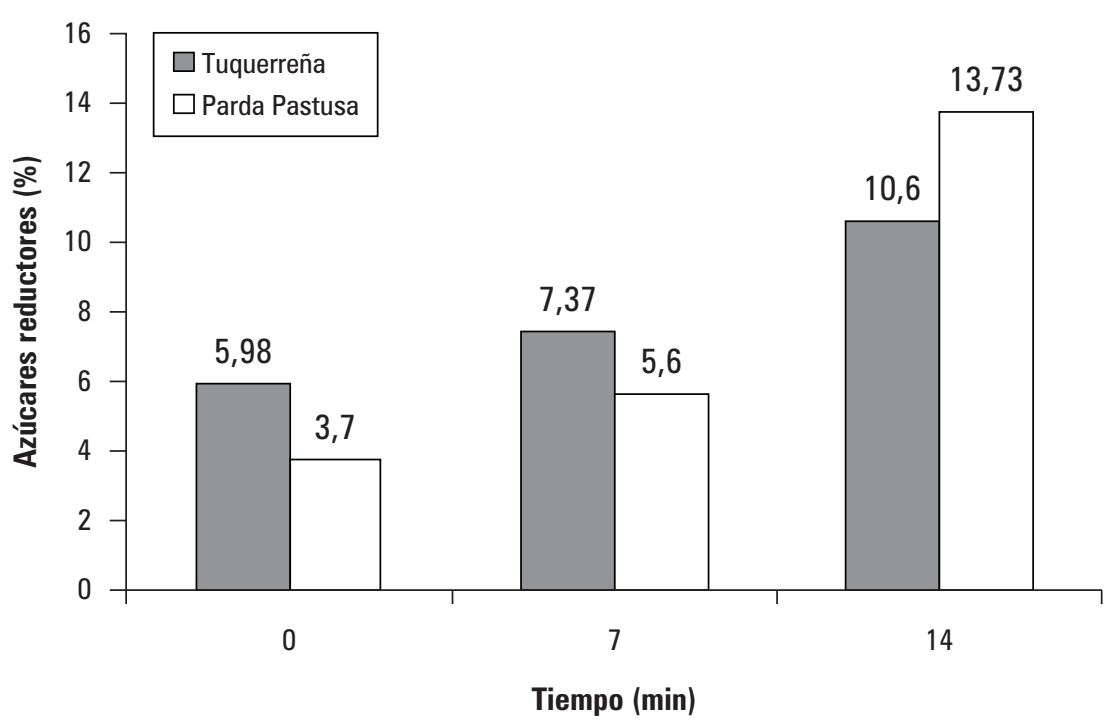

Figura 3. Sacarificación por acción de la enzima glucoamilasa.

Tabla 1. Caracterización del jarabe de glucosa.

\begin{tabular}{|c|c|c|c|}
\hline \multirow{2}{*}{ Análisis } & \multicolumn{2}{|c|}{ Variedad } & \multirow{2}{*}{ Técnica de análisis } \\
\hline & Parda Pastusa & Tuquerreña & \\
\hline Acidez (\% ácido láctico) & 0,45 & 0,34 & \multirow{2}{*}{${ }^{*}$ ISI 11-1e Acid Factor of Starch } \\
\hline $\mathrm{pH}$ & 4,54 & 4,42 & \\
\hline$\%$ de sólidos solubles totales ( ${ }^{\circ}$ Brix) & 37,8 & 35 & Método refractómetro \\
\hline Viscosidad (Cps) & 105 & 88,8 & $\begin{array}{l}\text { *ISI. 2002. Determination of viscosity of } \\
\text { starch by Brookfield. ISI 17-1e. En: Laboratory } \\
\text { methods. Science Park, Aarhus, Dinamarca, } \\
\text { International Starch Institute (ISI). }\end{array}$ \\
\hline \% de azúcares reductores & 13,01 & 10,6 & $\begin{array}{l}{ }^{*} \text { ISI 27. Reducing power by Lane and Eynon } \\
\text { method }\end{array}$ \\
\hline$\%$ equivalente de dextrosa (ED) & 30,96 & 34,85 & $\begin{array}{l}\text { ISO 5377:1981 Starch hydrolysis products -- } \\
\text { Determination of reducing power and dextrose } \\
\text { equivalent -- Lane and Eynon constant titre } \\
\text { method }\end{array}$ \\
\hline
\end{tabular}

* Normas ISI: disponibles en http://www.starch.dk/isi/methods/index.htm 
Los hidrolizados obtenidos corresponden a un líquido poco viscoso de color amarillo claro cristalino de sabor y olor característico. El porcentaje de sólidos solubles totales ( ${ }^{\circ}$ Brix) presentes en los jarabes de glucosa son de baja viscosidad frente a los valores estándar recomendados para este tipo de productos, según la ficha técnica de la enzima glucoamilasa. Este resultado se debe a que son jarabes no concentrados, reportando una humedad del 62,70\% para 'Parda Pastusa' y $65,75 \%$ para 'Tuquerreña', así como también a las características de las materias primas de donde provienen los almidones. Así mismo, como se observa en la tabla 1, el rendimiento y la eficacia del porcentaje logrado de equivalente de dextrosa fue mayor en el hidrolizado obtenido de la variedad Tuquerreña 34,85\% frente al valor de la Parda Pastusa de 30,96\% respectivamente. Al ser comparados estos valores con el estándar mínimo recomendado para un jarabe de glucosa muestran un porcentaje bajo, de acuerdo a lo reportado en la ficha técnica comercial, se indica que el valor mínimo es de 58\%. Estos resultados, se deben posiblemente a la no concentración del hidrolizado, dado que no se eliminó el agua.
En la figura 4 se observa la concentración de sólidos solubles totales que presentó el mosto y el comportamiento cinético frente a la actividad de producción de etanol por el microorganismo. Tanto la variedad Parda Pastusa como Tuquerreña demandaron un tiempo máximo de $32 \mathrm{~h}$ para la producción de bioetanol, así mismo se pudo observar que se realiza el proceso deforma exponencialmente a partir de las $16 \mathrm{~h}$.

Así mismo, en la figura 4 se reconoce que al inicio del proceso de fermentación el consumo de azúcares reductores es lento, coincide con el inicio del crecimiento de microorganismos y donde la producción de alcohol es muy baja. Esto se debe a que al inicio del proceso hay una reducción de los sólidos solubles totales debido a que la Saccharomyces cerevisiae convierte a la hexosas como la glucosa en alimento, y a su vez da inicio a la conversión del excedente en alcohol etílico y, por tanto, disminuye la concentración de sólidos solubles en el mosto.

De igual forma en la figura 5 , se observa la concentración de alcohol como se incrementa en

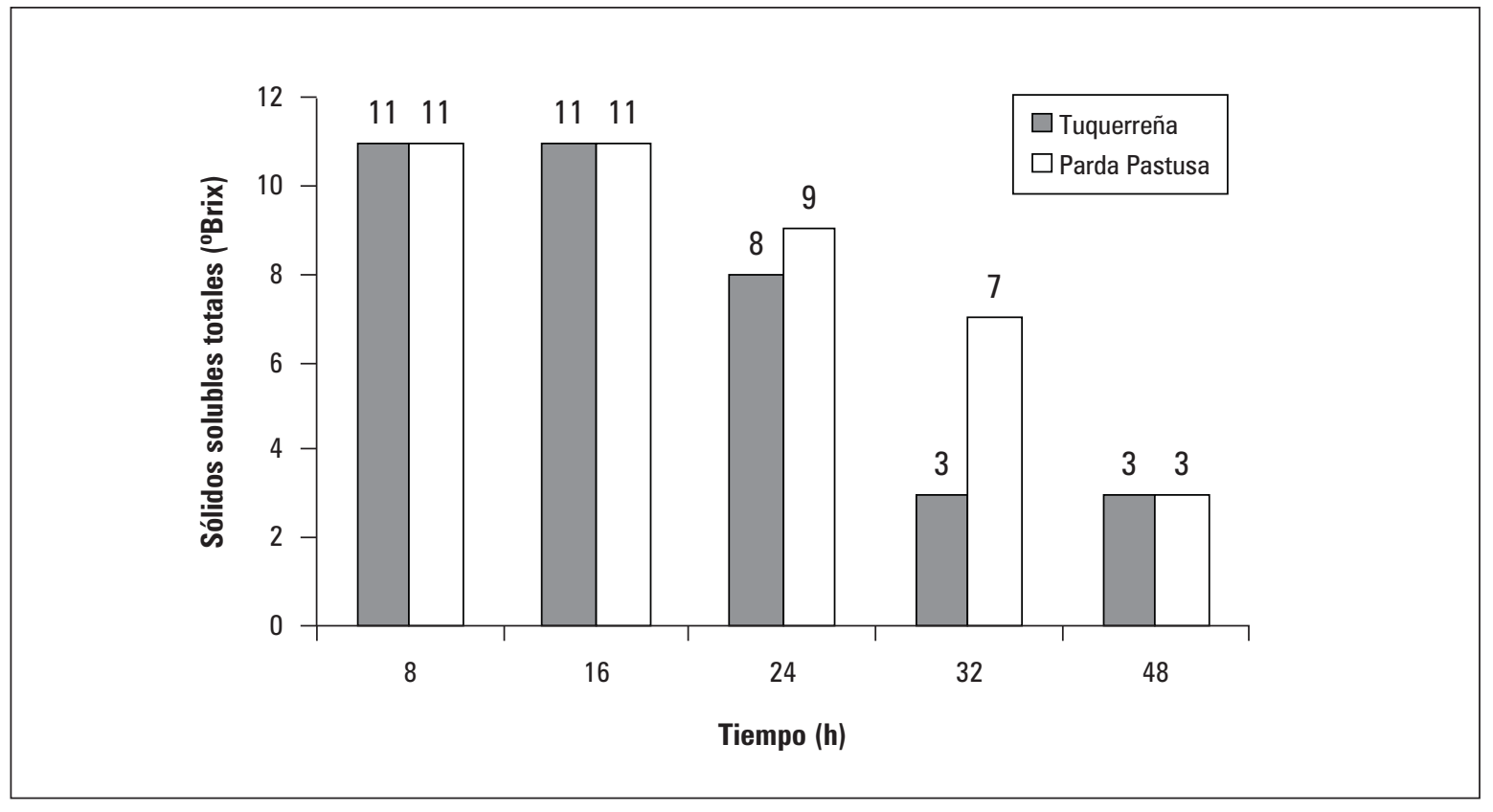

Figura 4. Sacarificación por acción de la enzima glucoamilasa. 


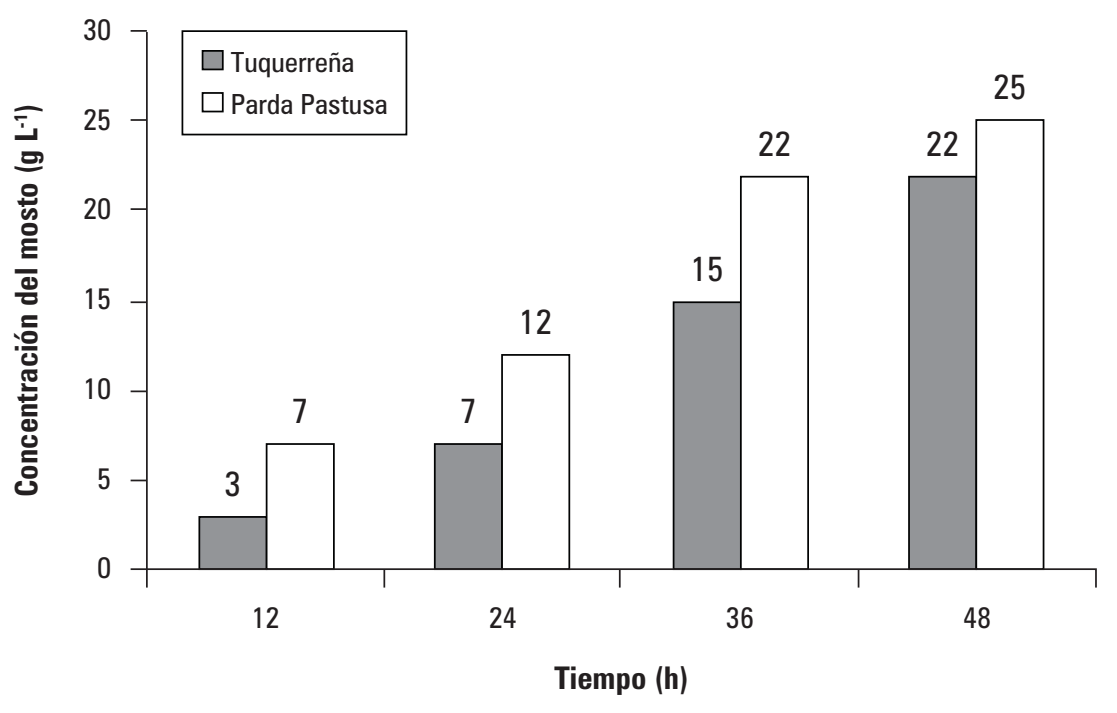

Figura 5. Concentración del mosto y variación de la concentración de bioetanol obtenido en el tiempo para las dos variedades.

función del tiempo de fermentación, evidenciando una disminución de la disponibilidad de sólidos solubles presentes en el mosto.

El producto resultante de la fermentación contiene entre $10 \%$ y $12 \%$ de etanol, así como residuos propios del proceso. Posteriormente, se destiló empleando una columna de destilación diferencial. Seguidamente, se evaluó la pureza del etanol obtenido, valor que se estima al $95,6 \%$ en volumen.

Para establecer si el etanol proveniente de la separación presentaba las características propias, se realizó una curva de calibración. La densidad del etanol obtenido fue de $1,014 \mathrm{~g} \mathrm{~cm}^{-3}$ a $20^{\circ} \mathrm{C}$ con una pureza del $94 \%$ que, comparado con lo reportado en la literatura $0,84 \mathrm{~g} \mathrm{~cm}^{-3}$ para el etanol es del 95,6\% (López, 2011), evidencia que no es puro. Esto se debe al sistema de separación empleado y posiblemente a que contienen otros productos de destilación, el cual no es eficiente dado que es una mezcla azeotropica (López, 2011). Es decir, una vez que se encuentra en una concen- tración de 95,5\% etanol/agua, los coeficientes de actividad del agua y del etanol son iguales, por lo que la concentración del vapor de la mezcla también es de 95,5\% etanol/agua y, por tanto, las destilaciones posteriores son ineficientes.

De acuerdo a lo anterior, se produjo 81,85 L de etanol por tonelada de papa con una concentración del $42,5 \% \mathrm{~m} / \mathrm{v}$ de sustrato, por lo que se infiere que el rendimiento es bajo al ser comparado con los rendimientos de 94,5 L de etanol/tonelada de papa, empleando una concentración del $20 \% \mathrm{~m} / \mathrm{v}$ de sustrato, reportado por González y Molina (2006). Sin embargo, es importante relacionar los resultados obtenidos con antelación dado que, no solo el método de destilación empleado incidió en el valor obtenido, sino que está claramente relacionado con la genética del tubérculo, sistema de producción y la zona geográfica, los cuales están directamente relacionadas con el contenido amilosa/amilopectina, parámetros que determinan directamente la cantidad de etanol obtenido, como se reporta en otras investigaciones (Gunaratne y Hoover, 2002). 
El rendimiento experimental varía entre $90 \%$ y 95\% del teórico, es decir, de 0,469 a 0,485 p/p. Los rendimientos en la industria varían entre 87\% y 93\% del rendimiento teórico (Boudarel, 1984). Teniendo en cuenta lo anterior y comparado con los resultados se puede afirmar que el etanol producido bajo condiciones de laboratorio es altamente puro pues el porcentaje está dentro del rango al cual se obtiene industrialmente.

\section{Análisis económico}

Determinado el costo e identificada cada actividad que intervino en el proceso, se configuró el costo total del producto, siguiendo el sistema ABC, como se observa en la tabla 2.

De acuerdo a la tabla 2 los costos indirectos representan la mayor proporción del costo total $(36,33 \%)$, debido a la cantidad de reactivos necesarios para control de la temperatura, el pH y el proceso de fermentación. Así mismo, el mayor costo en el proceso se da en la etapa de filtración, seguido de la cuantificación de azucares. Los materiales directos representan un 32,59\% del costo total. Este resultado a nivel de laboratorio corrobora lo obtenido en otras investigaciones, en las cuales se emplearon otras materias primas, como la caña de azúcar y maíz, y en donde representa el $60 \%$ de los costos del proceso hallado por Montoya et al. (2012).

Teniendo en cuenta lo señalado, se puede inferir que los componentes que inciden directamente sobre los rendimientos de la producción de alcohol son: la materia prima, el proceso de extracción y la acción de las enzimas durante el proceso de hidrólisis, dichos factores corroboran los hallados por González et al. (2006)

En el marco del análisis económico de la investigación se tomó el $20 \%$ de la producción total de papa, con la cual se estima que se pueden obtener 8.324.733 L, lo que arrojaría unos ingresos por 183.144.126.000 millones de pesos, tal como lo muestra la tabla 3.

Tabla 2. Estructura de costos según el sistema $A B C$ e ingresos para la producción de un litro de etanol a escala de laboratorio.

\begin{tabular}{|c|c|c|c|}
\hline \multicolumn{3}{|c|}{ Estructura de costos } & Porcentaje de participación (\%) \\
\hline Inventario inicial de materia prima & & 0 & \multirow{9}{*}{32,59} \\
\hline Compras de materia prima & $\$$ & $2.142,84$ & \\
\hline Materia prima disponible & $\$$ & $2.142,84$ & \\
\hline Inventario final de materia prima & $\$$ & $3.904,56$ & \\
\hline Costos materia prima utilizada & $\$$ & $6.047,40$ & \\
\hline \multicolumn{3}{|l|}{ Materiales indirectos } & \\
\hline \multicolumn{3}{|l|}{ Costo de material directo } & \\
\hline Mano de obra & $\$$ & $5.768,75$ & \\
\hline Mano de obra indirecta & & & \\
\hline Mano de obra directa & $\$$ & $5.768,75$ & \multirow[t]{6}{*}{31,08} \\
\hline Materiales indirectos & $\$$ & $4.242,62$ & \\
\hline \multicolumn{3}{|l|}{ Mano de obra } & \\
\hline Otros costos indirectos & $\$$ & $2.500,00$ & \\
\hline Costos indirectos de fabricación & $\$$ & $6.742,62$ & \\
\hline Costos del periodo & & $18.558,77$ & \\
\hline \multicolumn{4}{|c|}{ Estado de ingresos } \\
\hline Ingresos operacionales $(\$ / L)$ & $\$$ & $22.000 .00^{1}$ & \\
\hline Costo de ventas & $\$$ & $18.558,77$ & \\
\hline Utilidad bruta operacional & $\$$ & $3.341,23$ & \\
\hline Margen de contribución & & $\%$ / L etanol & \\
\hline
\end{tabular}

\footnotetext{
${ }^{1}$ Precio de venta en el mercado en Bogotá a 2012.
} 
Tabla 3. Estimación de la producción de bioetanol.

\begin{tabular}{|c|c|c|c|}
\hline Estado de resultados & Pesos/litro ${ }^{1}$ & Producción alcohol (L) & Producción total \\
\hline Ingresos operacionales $(\$ / L)$ & $\$ 22.000,00$ & \multirow{3}{*}{8.324 .733} & $183.144 .126 .000,00$ \\
\hline Costo de producción & $\$ 18.558,77$ & & 154.496.805.058,41 \\
\hline Utilidad bruta operacional & $\$ 3.341,23$ & & $28.647 .320 .941,59$ \\
\hline Margen de contribución: por litro de etanol & \multicolumn{3}{|c|}{$18,5 \%$} \\
\hline Margen de utilidad total & \multicolumn{3}{|c|}{$15,6 \%$} \\
\hline
\end{tabular}

Observando los datos de tabla 3 , es evidente que mientras la utilidad a partir de la producción de alcohol es del 15,6 \%, el productor de papa en fresco obtiene una pérdida alrededor del 10\%. Así mismo, sobresale el margen de comercialización que se obtiene de la papa en fresco que es del $42,98 \%$ y no el supuesto. Según los resultados, se infiere que a pesar de que los costos de la producción de alcohol son mayores que los costos de la papa en fresco, el margen de utilidad es mayor por la producción de alcohol, lo cual se visualiza en la tabla 4.

Según este análisis económico, es evidente que la alternativa de la producción de alcohol puede resultar como opción agroindustrial, siendo una oportunidad para el sector. Sin embargo, es importante aclarar que estas variedades no son las ideales para la producción de alcohol, dado que no se obtiene un rendimiento excelente en la extracción del almidón, como ilustra la figura 6 el punto de equilibrio se logra al producir 1.379 unidades. Esto indica que desde el punto de vista operacional, se alcanza el punto de equilibrio con un número relativamente bajo de unidades, que frente a la magnitud de las ventas de 8.324.733 litros, se está ante un proyecto favorable.

El análisis de sensibilidad de inversión, se realiza sobre tres escenarios de inversión por lo que para el cálculo del valor presente neto (VPN) y la tasa interna de retorno (TIR) se tomaron como inversión hipotética valores de \$100 millones, \$200 millones y $\$ 300$ millones, que se compara con una tasa de oportunidad del 10\%, como mínimo para una inversión en condiciones normales de una economía.

El análisis muestra que las tres propuestas son positivas, sin embargo, con una inversión inicial de $\$ 300$ millones, como lo muestra la tabla 5 , se requiere de un análisis más detallado en lo que respecta al VPN debido a que es menor que la inversión inicial, pero en términos generales el proyecto se presenta factible, frente a la tasa de oportunidad que se espera en el mercado si no se realizara la inversión.

Tabla 4. Estimación de ingresos y gastos en la producción de bioetanol.

\begin{tabular}{|c|c|c|c|c|}
\hline \multirow[b]{2}{*}{ Ítems } & \multicolumn{4}{|c|}{ Ingresos y gastos } \\
\hline & $\begin{array}{c}\text { Precios alcohol } \\
(\$ / \mathrm{L})\end{array}$ & $\begin{array}{l}\text { Precios totales de la papa } \\
\text { en fresco al productor } \\
(\$)\end{array}$ & $\begin{array}{c}\text { Precio total de venta de la } \\
\text { papa en fresco al consumidor } \\
(\$)\end{array}$ & $\begin{array}{c}\text { Margen } \\
\text { de comercialización } \\
\text { de la papa en fresco }\end{array}$ \\
\hline Ingresos & 183.144 .126 .000 & 79.359 .485 .800 & 113.465 .396 .583 & \multirow{3}{*}{34.105 .910 .783} \\
\hline Costos & 154.496 .805 .058 & 87.321 .400 .800 & & \\
\hline Utilidad & 28.647.320.942 & -7.961 .915 .000 & & \\
\hline Utilidad & $15,60 \%$ & $-10,00 \%$ & & $42,98 \%$ \\
\hline
\end{tabular}




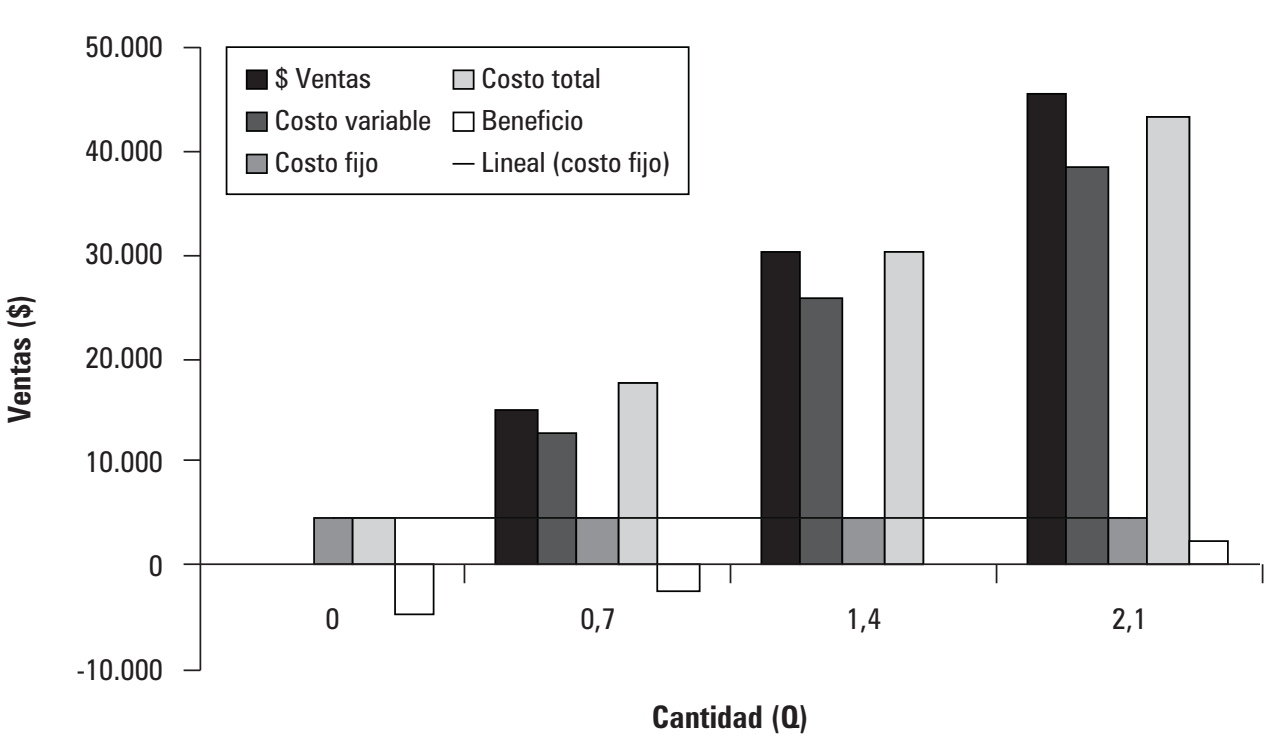

Figura 5. Punto de equilibrio de la producción de bioetanol.

Tabla 5. Flujo de caja para el escenario de inversión C de la producción de bioetanol.

\begin{tabular}{|c|c|c|c|c|c|c|}
\hline \multirow{2}{*}{ Ítem } & \multicolumn{6}{|c|}{ Años } \\
\hline & 0 & 1 & 2 & 3 & 4 & 5 \\
\hline Ingresos & & 183.144 .126 .000 & 192.118.188.174 & 200.763 .506 .642 & 209.797.864.441 & 219.238.768.341 \\
\hline Costos & 300.000 .000 & 154.496 .805 .058 & 159.131 .709 .210 & 163.905 .660 .486 & 168.822 .830 .301 & 173.887 .515 .210 \\
\hline Flujo neto & $(300.000 .000)$ & 28.647 .320 .942 & 32.986 .478 .964 & 36.857 .846 .155 & 40.975 .034 .140 & 45.351 .253 .130 \\
\hline VPN & & 237.062 .684 & & & & \\
\hline
\end{tabular}

De otra parte, el análisis de la TIR, en los tres escenarios de inversión y tomando de referencia para la comparación el 30\%, arroja como resultado valores que no son iguales al VPN, lo que permite concluir la alta rentabilidad del proyecto frente a tasa de oportunidad del 10\%, como lo muestra la tabla 6.

Tabla 6. Indicadores de rentabilidad para el escenario de inversión C de la producción de bioetanol.

\begin{tabular}{|c|c|c|c|c|c|c|}
\hline \multirow{3}{*}{ Ítem } & \multicolumn{6}{|c|}{ Flujos de Caja } \\
\hline & \multicolumn{6}{|c|}{ Años } \\
\hline & 0 & 1 & 2 & 3 & 4 & 5 \\
\hline Ingresos & & 183.144 .126 .000 & 192.118.188.174 & 200.763 .506 .642 & 209.797.864.441 & 219.238.768.341 \\
\hline Costos & 300.000 .000 & 154.496 .805 .058 & 159.131.709.210 & 163.905 .660 .486 & 168.822 .830 .301 & 173.887 .515 .210 \\
\hline Flujo neto & $(300.000 .000)$ & 28.647.320.942 & 32.986 .478 .964 & 36.857 .846 .155 & 40.975 .034 .140 & 45.351 .253 .130 \\
\hline VPN & & 237.062 .684 & & & & \\
\hline $\mathrm{TIR}$ & & $95,64 \%$ & & & & \\
\hline $\begin{array}{l}\text { Tasa de } \\
\text { descuento }\end{array}$ & & $10 \%$ & & & & \\
\hline
\end{tabular}




\section{CONCLUSIONES}

Se caracterizó el almidón de papa proveniente de las variedades Parda Pastusa y Tuquerreña, el mayor porcentaje de azúcares reductores se logra a una concentración de $0,672 \mathrm{~mL} \mathrm{~kg}^{-1}$ sustrato de enzima en solución al 42,2\% de almidón, empleando un tiempo de hidrólisis para la $\alpha$-amilasa de 30 min y para la glucoamilasa de 14 h. Así mismo, la condición de operación del enzima $\alpha$-amilasa es de un $\mathrm{pH}$ de 4,7 y una temperatura de $55^{\circ} \mathrm{C}$, alcanzando una máxima actividad y para la glucoamilasa, el pH óptimo es de 4,7 con una actividad equivalente al 70\% de la actividad máxima, y a una temperatura óptima es de $75^{\circ} \mathrm{C}$.

En el proceso de la fermentación alcohólica se obtuvo un consumo de azúcares reductores del 91\%, con una densidad del etanol de $1,014 \mathrm{~g} \mathrm{~mL}^{-1}$ a $20^{\circ} \mathrm{C}$ y una pureza del $94 \%$. La concentración inicial de sólidos para una eficiente gelatinización del almidón de papa fue del 42,68\%. Este proceso

\section{$\square$ \\ Alvis, A., C.A. Vélez, H.S. Villada y M. Rada-Mendo- za. 2008. Análisis fisico-químico y morfológico de almidones de ñame, yuca y papa y determina- ción de la viscosidad de las pastas. Información Tecnológica 19(1), 19-28. Doi: 10.4067/S0718- 07642008000100004}

Arias, L.M. 2014. Agricultura para la sostenibilidad. Revista RS 64, 50-51.

Bai, F., W. Anderson y M. Moo-Young. 2008. Ethanol fermentation technologies from sugar and starch feedstocks. Biotechnol. Adv. 26(1), 89-105. Doi: 10.1016/j.biotechadv.2007.09.002

Boudarel, M. 1984. Contribution á l'étude de la fermentation alcoolique á partir de jus de betteraves avec. Saccharomyces cerevisiae. Tesis de doctorado. Université de Bourgogne, Dijon, Francia.

Cano, J. y C. Naranjo, C. 2002. Obtención de etanol a partir de bano de rechazo por acción de Zymomonas mostró en la investigación un consumo de azúcares reductores del 41,6\% para el sustrato de la variedad Parda Pastusa y un $75 \%$ para el sustrato de la variedad Tuquerreña, lo cual tiene efecto directo en la producción de alcohol, donde se alcanza un $8,51 \% \mathrm{v} / \mathrm{v}$. De acuerdo con los resultados se produce $81,85 \mathrm{~L}$ de etanol/tonelada de papa con una concentración del $42,5 \% \mathrm{~m} / \mathrm{v}$ de sustrato.

Los costos que inciden en mayor proporción para la producción de etanol corresponden a la materia prima, debido al bajo porcentaje de extracción de almidón el cual corresponde al 18,7\%.

La evaluación económica arrojó que los costos de la producción de alcohol son mayores que los costos de la producción de papa. Sin embargo, el margen de utilidad es mayor para la producción de alcohol siendo del 15\% frente a la percibida en el proceso de la comercialización, donde hay una pérdida alrededor del 10\%. Así mismo, es importante destacar que el margen de intermediación en la comercialización de la papa es más del 40\%.

\section{REFERENCIAS BIBLIOGRÁFICAS}

mobilis. Trabajo de grado. Facultad de Ingeniería, Universidad Nacional de Colombia, Medellin, Colombia.

Cara, C., E. Ruiz, J. Oliva, F. Sáez y E. Castro. 2008. Conversion of olive tree biomass into fermentable sugars by dilute acid pretreatment and enzymatic hydrolisis. Bioresour. Technol. 99, 1869-1876. Doi:10.1016/j.biortech.2007.03.037

Castaño, H. y C. Mejía. 2008. Ethanol production from cassava starch using the process strategy simultaneous saccharification-fermentation. Vitae 15(2), 251-258

Castaño, P., B. Cardona, G.C. Mejía y C. Acosta. 2011. Producción de etanol a partir de harina de yuca en un sistema de hidrólisis enzimática y fermentación simultánea. Dyna 169, 158-166.

Fedebiocombustibles. 2015. Los biocombustibles y su aporte con el medio ambiente. Revista Nacional de Agricultura 964, 49-52. 
Fedepapa. 2010. Acuerdo de competitividad de la cadena agroalimentaria de la papa en Colombia. En: http://www.fedepapa.com/wp-content/uploads/ pdf/acuerdo-competitividad-cadena-agroalimentario; consulta: marzo de 2011.

González, J. y M. Molina. 2006. Estudio de los factores que afectan la hidrólisis enzimática y el proceso fermentativo para la producción de alcohol a partir de papa (Solanum tuberosum). Ingeniería 16(1), 29-39.

Gunaratne, A. y R. Hoover. 2002. Effect of heat- moisture treatment on the structure and physicochemical properties of tuber and root starches. Carbohydr. Polym. 49, 425-437. Doi: 10.1016/ S0144-8617(01)00354-X

Guzmán, F. 2008. Preparación y evaluación de proyectos de la industria química. Editorial Universidad Nacional de Colombia, Bogotá.

Kaplan, R.S. y S.R. Anderson. 2004. Time-driven activity based costing. Harvard Business Review 82(11), 131-138.

Lezame, S. 2006. Determinación a escala de laborario de las variables más importantes para la obtención de etanol a partir de almidón de yuca. Trabajo de grado. Universidad Autónoma de Occidente, Pereira, Colombia.

Lideboom, N., P. Chang y R. Tyler. 2004. Analytical, biochemical and physicochemical aspects of starch granule size, white os small granule starches. A review. Starch/Stärke 56(3-4), 89-99. Doi: 10.1002/ star.200300218

Lizarazo H., S.P., G.G. Hurtado R. y L.F. Rodríguez C. 2015. Physicochemical and morphological characterization of potato starch (Solanum tuberosum L.) as raw material for the purpose of obtaining bioethanol. Agron. Colomb. 33(2). Doi: 10.15446/ agron.colomb.v33n2.47239

López, S. A. 2011. Proyecto de implementación de un sistema de producción a gran escala, de etanol al $70 \%$ peso/peso, a partir de una dilución de etanol al 95\% peso/peso, en una empresa en la ciudad de
Guatemala. Tesis de maestría. Facultad de Ciencias Químicas y Farmacia, Universidad de San Carlos de Guatemala, Guatemala.

Miranda, J.J. 2012. Gestión de proyectos: identificación, formulación y evaluación. MM Editores, Bogotá.

Montoya, M.I., J.A. Quintero, O.J. Sanchez y C.A. Cardona. 2012. Evaluación económica del proceso de obtención de alcohol carburante a partir de caña de azúcar y maíz. Revista Universidad Eafit 41(139), 76-87.

Moreno, J. D., M.d.S. Cerón, J.L. Zapata y L.A. Peña. 2006. Corpoica - Mary: variedad de papa mejorada de alto rendimiento para consumo en fresco y procesamiento en hojuelas. Innovación y Cambio Tecnológico 4(4), 1-2.

Nústez, C. 2011. Variedades colombianas de papa. Red Latin Papa Fontagro Inia España, Grupo Imágenes, Universidad Nacional de Colombia. Bogotá.

Sánchez, O.J. y C.A. Cardona. 2007. Producción de alcohol carburante, una alternativa para el desarrollo agroindustrial. Facultad de Ingeniería y Arquitectura, Universidad Nacional de Colombia, Manizales, Colombia.

Santis-Espinosa, L.F., B.Y. Perez-Sariñana, C.A. Guerrero-Fajardo, S. Saldaña-Trinidad, E.C. Lopéz-Vidaña y P. J. Sebastian. 2015. Drying mango (Mangifera indica L.) with solar energy as a pretreatment for bioethanol production. BioResources 10(3), 6044-6054. Doi: 10.15376/biores.10.3.6044-6054

Singh, J. y N. Singh. 2001. Studies on the morphological, thermal and rheological properties of starch separated from some Indian potato cultivars. Food Chem. 75, 67-77. Doi: 10.1016/S03088146(01)00189-3

Wingrove, A. y R. Caret. 1981. Química orgánica. Ed. Harla, México.

Zuleta, L.A. y L. Jaramillo. 2000. La industria de licores en Colombia. Estudio elaborado para la industria internacional. Fedesarrollo, Bogotá. 